PROCEEDINGS OF THE

AMERICAN MATHEMATICAL SOCIETY

Volume 139, Number 8, August 2011, Pages 3009-3014

S 0002-9939(2011)10729-9

Article electronically published on January 13, 2011

\title{
SYMPLECTIC REAL BOTT MANIFOLDS
}

\author{
HIROAKI ISHIDA \\ (Communicated by Jon G. Wolfson)
}

\begin{abstract}
A real Bott manifold is the total space of an iterated $\mathbb{R} P^{1}$-bundle over a point, where each $\mathbb{R} P^{1}$-bundle is the projectivization of a Whitney sum of two real line bundles. In this paper, we characterize real Bott manifolds which admit a symplectic form. In particular, it turns out that a real Bott manifold admits a symplectic form if and only if it is cohomologically symplectic. In this case, it admits even a Kähler structure. We also prove that any symplectic cohomology class of a real Bott manifold can be represented by a symplectic form. Finally, we study the flux of a symplectic real Bott manifold.
\end{abstract}

\section{INTRODUCTION}

A real Bott tower (of height $n$ ) is a sequence of $\mathbb{R P}^{1}$-bundles:

$$
M_{n} \rightarrow M_{n-1} \rightarrow \cdots \rightarrow M_{1} \rightarrow M_{0}=\{\text { a point }\},
$$

where each $\mathbb{R P}^{1}$-bundle $M_{i} \rightarrow M_{i-1}$ is the projectivization of a Whitney sum of two real line bundles on $M_{i-1}$. Each $M_{i}$ is called a real Bott manifold. Clearly $M_{1}=\mathbb{R P}^{1}$ and $M_{2}=\left(\mathbb{R P}^{1}\right)^{2}$ or a Klein bottle. If every bundle in the tower is trivial, then $M_{n}=\left(\mathbb{R P}^{1}\right)^{n}$. However, there are many choices of non-trivial bundles at each stage in the tower, and it is known that there are many different diffeomorphism classes in real Bott manifolds ([7, [5]). A real Bott manifold is also an example of a real toric manifold which admits a flat Riemannian metric ([7]).

Although orientable real Bott manifolds occupy a small portion in all real Bott manifolds (4]), the number of orientable ones of dimension $n$ approaches infinity as $n$ approaches infinity. Among those orientable ones, some are symplectic, i.e., admit a symplectic form. In this paper we give a complete characterization of symplectic real Bott manifolds (Theorem 3.1). In particular, we prove that among real Bott manifolds $M$ the following are equivalent:

(1) $M$ is cohomologically symplectic,

(2) $M$ is symplectic,

(3) $M$ admits a Kähler structure.

We call a $2 n$-dimensional closed manifold $M$ cohomologically symplectic if there is a cohomology class $\alpha \in \mathrm{H}^{2}(M ; \mathbb{R})$ such that $\alpha^{n} \neq 0$. We remark that the implication $(3) \Rightarrow(2) \Rightarrow(1)$ always holds, but the reverse implications $(1) \Rightarrow(2)$ and $(2) \Rightarrow(3)$ do not hold in general, as is well-known. For example, $\mathbb{C} P^{2} \# \mathbb{C} P^{2}$ is

Received by the editors January 19, 2010 and, in revised form, July 29, 2010.

2010 Mathematics Subject Classification. Primary 57R17, 57S25.

Key words and phrases. Toric topology, symplectic topology, real Bott manifold.

(C)2011 American Mathematical Society

Reverts to public domain 28 years from publication 
cohomologically symplectic but not symplectic because it does not admit an almost complex structure, and a certain $T^{2}$-bundle over $T^{2}$ constructed in 9 is symplectic but does not admit a Kähler structure.

This paper is organized as follows. In Section 2 we recall the quotient description of real Bott manifolds. In Section 3 we state and prove our main theorem. In Section 4 we study the flux group of a symplectic real Bott manifold.

Throughout this paper, all cohomology will be de Rham cohomology over $\mathbb{R}$.

\section{Quotient DESCRIPTION OF REAL BotT MANiFOLDS}

In this section, we recall the quotient description of real Bott manifolds (see 7 and [5] for details) and determine the cohomology ring of a real Bott manifold.

Let $\mathfrak{B}(n)$ be the the set of $n \times n$ upper triangular matrices whose entries are 0 's and 1's with zero diagonal entries. For a matrix $A \in \mathfrak{B}(n), A_{j}^{i}$ denotes the $(i, j)$ entry of $A$, and $A^{i}$ (respectively, $A_{j}$ ) denotes the $i$-th row (respectively, the $j$-th column) of $A$. Let $S^{1}$ be the unit circle in $\mathbb{C}$. We then define the involution $a_{i}$ on $T^{n}:=\left(S^{1}\right)^{n}$ by

$$
a_{i}\left(z_{1}, \ldots, z_{n}\right):=\left(z_{1}, \ldots, z_{i-1},-z_{i}, z_{i+1}^{(-1)^{A_{i+1}^{i}}}, \ldots, z_{n}^{(-1)^{A_{n}^{i}}}\right)
$$

for $\left(z_{1}, \ldots, z_{n}\right) \in T^{n}$ and $i=1, \ldots, n$. Let $G(A)$ denote the transformation group on $T^{n}$ generated by the $a_{i}$ 's. Then the quotient space $M(A):=T^{n} / G(A)$ is known to be a real Bott manifold, and every real Bott manifold can be obtained as $M(A)$ for some $A \in \mathfrak{B}(n)$. The real Bott manifold $M(A)$ does not determine uniquely the matrix $A$; however, all geometrical information on $M(A)$ can be read from $A$. For example,

$$
M(A) \text { is orientable } \Longleftrightarrow \sum_{j=1}^{n} A_{j}^{i}=0 \text { in } \mathbb{Z} / 2 \text { for any } i
$$

(see [7]).

It is also helpful to describe $M(A)$ as the quotient of $\mathbb{R}^{n}$ by affine transformations. In fact, let $\Gamma(A)$ denote the affine transformation group on $\mathbb{R}^{n}$ generated by $s_{i}$ 's defined by

$$
s_{i}\left(u_{1}, \ldots, u_{n}\right):=\left(u_{1}, \ldots, u_{i-1}, u_{i}+\frac{1}{2},(-1)^{A_{i+1}^{i}} u_{i+1}, \ldots,(-1)^{A_{n}^{i}} u_{n}\right)
$$

for $i=1, \ldots, n$. Then, an exponential map from $\mathbb{R}$ to $S^{1}$ sending $u$ to $\exp (2 \pi \sqrt{-1} u)$ induces a diffeomorphism from $\mathbb{R}^{n} / \Gamma(A)$ onto $T^{n} / G(A)=M(A)$.

Let $d u_{1}, \ldots, d u_{n}$ denote the standard 1-forms on $\mathbb{R}^{n}$. Since each $d u_{j}$ is invariant under parallel translations on $\mathbb{R}^{n}$, it descends to a closed 1-form on $T^{n} \cong \mathbb{R}^{n} / \mathbb{Z}^{n}$, which we also denote by $d u_{j}$. The (de Rham) cohomology ring $\mathrm{H}^{*}\left(T^{n}\right)$ of $T^{n}$ is the exterior algebra in $n$ variables $\left[d u_{1}\right], \ldots,\left[d u_{n}\right]$ over $\mathbb{R}$, where $\left[d u_{j}\right]$ denotes the cohomology class represented by the 1 -form $d u_{j}$. It follows from (2.1) or (2.3) that the endomorphism $a_{i}^{*}$ of $\mathrm{H}^{*}\left(T^{n}\right)$ induced by $a_{i} \in G(A)$ is given by

$$
a_{i}^{*}\left(\left[d u_{j}\right]\right)= \begin{cases}{\left[d u_{j}\right]} & \text { if } A_{j}^{i}=0 \\ -\left[d u_{j}\right] & \text { if } A_{j}^{i}=1 .\end{cases}
$$

We note that since $M(A)=T^{n} / G(A)$ and $G(A)$ is a finite group, we have

$$
\mathrm{H}^{*}(M(A))=\mathrm{H}^{*}\left(T^{n}\right)^{G(A)}
$$


(see [3. Theorem 2.4 on p. 120], for example), where the right hand side denotes the $G(A)$-invariants in $\mathrm{H}^{*}\left(T^{n}\right)$.

Lemma 2.1. Let $J$ be a subset of $\{1, \ldots, n\}$. Then $\prod_{j \in J}\left[d u_{j}\right] \in \mathrm{H}^{*}\left(T^{n}\right)$ is $G(A)$ invariant if and only if $\sum_{j \in J} A_{j}=0$ in $\mathbb{Z} / 2$.

Proof. By (2.4), we have

$$
a_{i}^{*}\left(\prod_{j \in J}\left[d u_{j}\right]\right)=(-1)^{\sum_{j \in J} A_{j}^{i}} \prod_{j \in J}\left[d u_{j}\right] .
$$

Thus, $\prod_{j \in J}\left[d u_{j}\right]$ is fixed by $a_{i}^{*}$ if and only if $\sum_{j \in J} A_{j}^{i}=0$ in $\mathbb{Z} / 2$. This implies the lemma since $G(A)$ is generated by the $a_{i}$ 's.

\section{MAIN THEOREM}

The following is our main theorem in this paper.

Theorem 3.1. Let $A \in \mathfrak{B}(2 n)$. The following conditions are equivalent:

(1) $M(A)$ is cohomologically symplectic; that is, there exists an $\alpha \in \mathrm{H}^{2}(M(A))$ such that $\alpha^{n}$ is non-zero.

(2) There exist $n$ subsets $\left\{j_{1}, j_{n+1}\right\}, \ldots,\left\{j_{n}, j_{2 n}\right\}$ of $\{1,2, \ldots, 2 n\}$ such that

- $\coprod_{k}^{n}\left\{j_{k}, j_{k+n}\right\}=\{1,2, \ldots, 2 n\}$ and

- $A_{j_{1}}=A_{j_{n+1}}, \ldots, A_{j_{n}}=A_{j_{2 n}}$.

(3) There exists a symplectic form on $M(A)$.

(4) There exists a Kähler structure on $M(A)$.

Moreover, any $\alpha \in \mathrm{H}^{2}(M(A))$ in (1) can be represented by a symplectic form on $M(A)$.

Proof. Because any closed symplectic manifold is cohomologically symplectic and any Kähler manifold is a symplectic manifold, it suffices to prove implications (1) $\Rightarrow(2)$ and $(2) \Rightarrow(4)$.

Proof of $(1) \Rightarrow(2)$. Assume that there exists a de Rham cohomology class $\alpha \in \mathrm{H}^{2}(M(A))$ such that $\alpha^{n} \neq 0$. We identify $\mathrm{H}^{*}(M(A))$ with $\mathrm{H}^{*}\left(T^{n}\right)^{G(A)}$ by (2.5). Then it follows from Lemma 2.1 that we can write $\alpha$ uniquely as

$$
\alpha=\sum_{j<k, A_{j}=A_{k}} c_{j, k}\left[d u_{j} \wedge d u_{k}\right] \quad \text { with some } c_{j, k} \in \mathbb{R} .
$$

Thus $\alpha^{n} \neq 0$ implies condition (2).

Proof of $(2) \Rightarrow(4)$. Assume that $A \in \mathfrak{B}(2 n)$ satisfies condition (2), namely $A_{j_{k}}=A_{j_{k+n}}$ for $k=1, \ldots, n$. Then we identify $\mathbb{R}^{2 n}$ with $\mathbb{C}^{n}$ by

$$
z_{k}:=u_{j_{k}}+\sqrt{-1} u_{j_{k+n}}
$$

for $k=1, \ldots, n$. Consider the standard Hermitian metric on $\mathbb{C}^{n}$. Then, $\Gamma(A)$ acts on $\mathbb{C}^{n}$ as biholomorphisms and isometries. In fact, through the above identification, it follows from (2.3) that the action of $s_{i} \in \Gamma(A)$ on $\mathbb{C}^{n}$ is given by

$$
s_{i}\left(z_{1}, \ldots, z_{n}\right)_{k}= \begin{cases}z_{k}+\frac{1}{2} & \text { if } i=j_{k} \\ z_{k}+\frac{\sqrt{-1}}{2} & \text { if } i=j_{k+n} \\ z_{k} & \text { if } A_{j_{k}}^{i}=A_{j_{k+n}}^{i}=0 \text { and } i \neq j_{k}, j_{k+n} \\ -z_{k} & \text { if } A_{j_{k}}^{i}=A_{j_{k+n}}^{i}=1,\end{cases}
$$


where the left hand side denotes the $k$-th component of $s_{i}\left(z_{1}, \ldots, z_{n}\right)$. Thus the quotient $M(A)=\mathbb{C}^{n} / \Gamma(A)$ inherits the standard Kähler structure on $\mathbb{C}^{n}$.

Finally, we shall prove the last statement in the theorem. As observed above, $\alpha \in \mathrm{H}^{2}(M(A))$ is of the form (3.1). We then define the differential closed 2-form $\omega$ on $\mathbb{R}^{2 n}$ by

$$
\omega:=\sum_{j<k, A_{j}=A_{k}} c_{j, k} d u_{j} \wedge d u_{k} .
$$

Comparing (3.1) with (3.2), one sees that the condition $\alpha^{n} \neq 0$ implies that $\omega^{n}$ is nowhere zero. Thus $\omega$ is a symplectic form on $\mathbb{R}^{2 n}$. Since $\omega$ is invariant under the $\Gamma(A)$-action on $\mathbb{R}^{2 n}, \omega$ descends to a symplectic form on the quotient $M(A)=$ $\mathbb{R}^{2 n} / \Gamma(A)$, and this represents the given class $\alpha$.

Example 3.2. Let $A \in \mathfrak{B}(4)$. If $A$ is the zero matrix, then $M(A)$ is the 4dimensional torus and symplectic. Suppose that $A$ is non-zero and $M(A)$ is symplectic. Then it follows from Theorem 3.1 (2) that $A$ is one of the following:

$$
\left(\begin{array}{llll}
0 & 1 & 1 & 0 \\
0 & 0 & 0 & 0 \\
0 & 0 & 0 & 0 \\
0 & 0 & 0 & 0
\end{array}\right), \quad\left(\begin{array}{llll}
0 & 1 & 0 & 1 \\
0 & 0 & 0 & 0 \\
0 & 0 & 0 & 0 \\
0 & 0 & 0 & 0
\end{array}\right), \quad\left(\begin{array}{llll}
0 & 0 & 1 & 1 \\
0 & 0 & 0 & 0 \\
0 & 0 & 0 & 0 \\
0 & 0 & 0 & 0
\end{array}\right), \quad\left(\begin{array}{llll}
0 & 0 & 1 & 1 \\
0 & 0 & 1 & 1 \\
0 & 0 & 0 & 0 \\
0 & 0 & 0 & 0
\end{array}\right),\left(\begin{array}{llll}
0 & 0 & 0 & 0 \\
0 & 0 & 1 & 1 \\
0 & 0 & 0 & 0 \\
0 & 0 & 0 & 0
\end{array}\right) .
$$

Real Bott manifolds $M(A)$ for $A$ above are diffeomorphic to each other but not diffeomorphic to the 4-dimensional torus ([7, [5]). One sees that $M(A)$ is the total space of a non-trivial orientable $T^{2}$-bundle over $T^{2}$. Note that total spaces of orientable $T^{2}$-bundles over $T^{2}$ are symplectic and classified in $\underline{6}$.

Now we determine the type of $M(A)$. Suppose

$$
A=\left(\begin{array}{llll}
0 & 0 & 1 & 1 \\
0 & 0 & 0 & 0 \\
0 & 0 & 0 & 0 \\
0 & 0 & 0 & 0
\end{array}\right)
$$

Then, $A_{1}=A_{2}$ and $A_{3}=A_{4}$. We identify $\mathbb{R}^{4}$ with $\mathbb{C}^{2}$ by

$$
\begin{aligned}
z_{1} & :=\frac{1}{2} u_{1}+\frac{\sqrt{-1}}{2} u_{2}, \\
z_{2} & :=\frac{1}{2} u_{3}+\frac{\sqrt{-1}}{2} u_{4} .
\end{aligned}
$$

Then, the action of $s_{1}, s_{2}, s_{3}$ and $s_{4}$ on $\mathbb{C}^{2}$ are given by

$$
\begin{gathered}
s_{1}\left(z_{1}, z_{2}\right)=\left(z_{1}+1,-z_{2}\right), \\
s_{2}\left(z_{1}, z_{2}\right)=\left(z_{1}+\sqrt{-1}, z_{2}\right), \\
s_{3}\left(z_{1}, z_{2}\right)=\left(z_{1}, z_{2}+1\right)
\end{gathered}
$$

and

$$
s_{4}\left(z_{1}, z_{2}\right)=\left(z_{1}, z_{2}+\sqrt{-1}\right) .
$$

Let $E$ denote the elliptic curve $\mathbb{C} / \mathbb{Z} \oplus \sqrt{-1} \mathbb{Z}$. Then,

$$
M(A)=\mathbb{C}^{2} / \Gamma(A) \cong \mathbb{C} \times E /\left\langle s_{1}, s_{2}\right\rangle .
$$

Hence our $M(A)$ is of type $\{-I, I,(0,0)\}$ in [6. Table 1] and is a hyper-elliptic surface of type (a1) in [2, p. 148].

Finally we note that if

$$
A=\left(\begin{array}{llll}
0 & 1 & 1 & 0 \\
0 & 0 & 1 & 1 \\
0 & 0 & 0 & 0 \\
0 & 0 & 0 & 0
\end{array}\right)
$$


then $M(A)$ is orientable by (2.2), but not symplectic. Therefore the class of symplectic real Bott manifolds is strictly smaller than that of orientable real Bott manifolds.

\section{THE FLUX GROUP}

In this section, we will study the flux group of a symplectic real Bott manifold. To do that, we recall the definition of a flux group for a general symplectic manifold.

Let $(M, \omega)$ be a closed symplectic manifold. A diffeomorphism $\phi: M \rightarrow M$ is called a symplectomorphism if $\phi^{*} \omega=\omega$ and the group of symplectomorphisms of $(M, \omega)$ is denoted by $\operatorname{Symp}(M, \omega)$. Associated to a smooth function $f: M \rightarrow \mathbb{R}$, the Hamiltonian vector field $X_{f}$ is defined by $i_{X_{f}} \omega=d f$. For a one-parameter family $\left\{f_{t}\right\}_{0 \leq t \leq 1}$ of functions, we obtain a one-parameter family $\left\{X_{f_{t}}\right\}_{0 \leq t \leq 1}$ of Hamiltonian vector fields, and integrating $\left\{X_{f_{t}}\right\}$, we obtain a one-parameter family $\left\{\phi_{t}\right\}_{0 \leq t \leq 1}$ of diffeomorphisms defined by

$$
\frac{d}{d t} \phi_{t}=X_{f_{t}} \circ \phi_{t} \text { and } \phi_{0}=\mathrm{id} \text {. }
$$

The time-one map $\phi_{1}$ is a symplectomorphism and is called a Hamiltonian diffeomorphism. It is known that all Hamiltonian diffeomorphisms of $(M, \omega)$ form a subgroup, denoted $\operatorname{Ham}(M, \omega)$, of the identity component $\operatorname{Symp}_{0}(M, \omega)$ of $\operatorname{Symp}(M, \omega)$. For a symplectic isotopy $\left\{\phi_{t}\right\}$, that is, an isotopy through symplectomorphisms, we obtain a one-parameter family $\left\{X_{t}\right\}$ of vector fields defined by

$$
\frac{d}{d t} \phi_{t}=X_{t} \circ \phi_{t}
$$

The flux of $\left\{\phi_{t}\right\}$ is then defined to be

$$
\int_{0}^{1}\left[i_{X_{t}} \omega\right] d t \in \mathrm{H}^{1}(M)
$$

It is known that the flux depends only on the homotopy class of symplectic isotopies with fixed end points $\phi_{0}=\mathrm{id}$ and $\phi_{1}$, so that it defines a homomorphism

$$
\text { Flux : } \operatorname{Symp}_{0}(M, \omega) \rightarrow \mathrm{H}^{1}(M) / \Gamma_{\omega},
$$

where $\Gamma_{\omega}$ is the image of the fundamental group $\pi_{1}\left(\operatorname{Symp}_{0}(M, \omega)\right)$ by the flux and is called the flux group of $(M, \omega)$. The solution of the flux conjecture $([8])$ says that the subgroup $\Gamma_{\omega}$ of $\mathrm{H}^{1}(M)$ is closed and discrete. According to [1, the kernel of Flux is exactly equal to $\operatorname{Ham}(M, \omega)$; in other words, we have an exact sequence

$$
\{1\} \rightarrow \operatorname{Ham}(M, \omega) \rightarrow \operatorname{Symp}_{0}(M, \omega) \stackrel{\text { Flux }}{\rightarrow} \mathrm{H}^{1}(M) / \Gamma_{\omega} .
$$

Now, we consider the flux of a symplectic real Bott manifold.

Theorem 4.1. Let $M(A)$ be a real Bott manifold with a symplectic form $\omega$ given by (3.2). Then, the flux group $\Gamma_{\omega}$ is a lattice group of $\mathrm{H}^{1}(M(A))$ of full rank.

Proof. It follows from Lemma 2.1 that $\mathrm{H}^{1}(M(A))$ is generated by $\left[d u_{j}\right]$ with $A_{j}=$ 0 , and since $M(A)$ is symplectic, the number of zero columns in $A$ is even by Theorem 3.1, so that $\mathrm{H}^{1}(M(A))$ is even dimensional. Let $2 r$ be the dimension of $\mathrm{H}^{1}(M(A))$. We may assume that $\mathrm{H}^{1}(M(A))$ is generated by $d u_{1}, \ldots, d u_{2 r}$ by changing the suffices of the coordinates. Moreover, through a linear coordinate 
change of the first $2 r$ coordinates $u_{1}, \ldots, u_{2 r}$, we may assume that the symplectic form $\omega$ on $M(A)$ is of the form

$$
\omega=\sum_{i=1}^{r} d u_{i} \wedge d u_{i+r}+\sum_{j<k, A_{j}=A_{k} \neq 0} c_{j, k} d u_{j} \wedge d u_{k} .
$$

Since $M(A)=T^{2 n} / G(A)$ and $A_{p}=0$ for $p=1, \ldots, 2 r$, the multiplication of $S^{1}$ on the $p$-th coordinate on $T^{2 n}$ for $1 \leq p \leq 2 r$ descends to an $S^{1}$-action on $M(A)$ and defines a symplectic isotopy $\left\{\phi_{t}^{p}\right\}$. The one-parameter family $\left\{X_{t}^{p}\right\}$ of vector fields associated with $\left\{\phi_{t}^{p}\right\}$ is then $\partial / \partial u_{p}$ (possibly up to a non-zero constant), so that it follows from (4.1) and (4.2) that

$$
\text { the flux of }\left\{\phi_{t}^{p}\right\}=\int_{0}^{1}\left[i_{X_{t}^{p}} \omega\right] d t=\int_{0}^{1}\left[d u_{q}\right] d t=\left[d u_{q}\right] \text {, }
$$

where $q=p+r$ if $1 \leq p \leq r$ and $q=p-r$ if $r+1 \leq p \leq 2 r$. This shows that $\Gamma_{\omega}$ spans $\mathrm{H}^{1}(M(A))$ over $\mathbb{R}$. Since $\Gamma_{\omega}$ is closed and discrete in $\mathrm{H}^{1}(M(A))$ as remarked before, it must be a lattice group of $\mathrm{H}^{1}(M(A))$ of full rank.

\section{ACKNOWLEDGMENTS}

The author would like to thank Professor Mikiya Masuda for a stimulating discussion about toric topology and symplectic topology. He also would like to thank Professor Kaoru Ono for explaining the flux group of a symplectic manifold, Professor Takahiko Yoshida for informing him of the paper [6], and Shintaro Kuroki and Yunhyung Cho for useful comments on the paper.

\section{REFERENCES}

[1] A. Banyaga, Sur la structure du groupe des difféomorphismes qui préservent une forme symplectique, Comment. Math. Helv. 53 (1978), 174-227. MR490874 (80c:58005)

[2] W. Barth, C. Peters and A. Van de Ven, Compact Complex Surfaces, Springer, 1984. MR749574 (86c:32026)

[3] G. E. Bredon, Introduction to Compact Transformation Groups, Pure and Applied Mathematics, 46, Academic Press, 1972. MR0413144 (54:1265)

[4] S. Choi, The number of orientable small covers over cubes, Proc. Japan Acad., 86, Ser. A (2010), 97-100.

[5] S. Choi, M. Masuda and S. Oum Classification of real Bott manifolds and acyclic digraphs, preprint, arXiv:math.AT/1006.4658.

[6] H. Geiges, Symplectic structures on $T^{2}$-bundles over $T^{2}$, Duke Math. J. 67 (1992), 539-555. MR:1181312 (93i:57036)

[7] Y. Kamishima and M. Masuda, Cohomological rigidity of real Bott manifolds, Alg. \& Geom. Top. 9 (2009), 2479-2502. MR 2576506

[8] K. Ono, Floer-Novikov cohomology and the flux conjecture, GAFA, Geom. Funct. Anal. 16 (2006), 981-1020. MR2276532 (2007k:53147)

[9] W. P. Thurston, Some simple examples of symplectic manifolds, Proc. Amer. Math. Soc. 55 (1976), 467-468. MR0402764(53:6578)

Graduate School of Science, Osaka City University, Sugimoto, Sumiyoshi-ku, Osaka 558-8585, JAPAN

E-mail address: hiroaki.ishida86@gmail.com 